into sequences, (2) a course table showing what teachers give and what pupils wish to take the various courses, (3) a conflict table showing the common members of each pair of courses, if their number is not more than a given number, say five, and, if more, showing that fact. The first of these tables is a unary operation table, the second a dyadic relation table, the third a combination of a binary operation table and a dyadic relation table.

The course table is cut into strips showing the class lists, which are used to form the conflict table. This table is then cut into strips, one for each course, which are then easily assembled into groups, so as to have as few conflicts as possible. The courses of any one group are placed at the same sequence of hours.

The form in which the schedule first appears is itself a dyadic relation table, the left-hand column containing the department names, the top row containing the numbers of the sequences, and the intersections of rows and columns the course numbers.

F. N. Cole, Secretary.

\title{
A NECESSARY AND SUFFICIENT CONDITION FOR THE UNIFORM CONVERGENCE OF A CERTAIN CLASS OF INFINITE SERIES.
}

BY DR. N. J. LENNES.

(Read before the American Mathematical Society, February 25, 1911.)

ALl numbers $x$ such that $a \leqq x \leqq b$ constitute the interval $a b$. A function $f(x)$ is said to be continuous on an interval $a b$ if (1) it is continuous in the ordinary sense for every value of $x, a<x<b,(2)$ if it has right hand continuity for $x=a$ and left hand continuity for $x=b$.

TheOREM. If on the interval $a b$

$$
\sum_{n=0}^{\infty} U_{n}(x)=f(x),
$$

$U_{i}(x)(i=0, \cdots, \infty)$ and $f(x)$ being continuous on the interval $a b$, then in order that $\sum_{n=0}^{\infty} U_{n}(x)$ shall be uniformly convergent on 
the interval $a b$ it is necessary and sufficient that for any $x_{i}$ on $a b$, any arbitrary number $\delta$ (however small) and any arbitrary integer $N$ there is an integer $N^{\prime}(i, \delta, N) *$ greater than $N$, which satisfies the following, condition:

On the interval $x_{i}-\delta, x_{i}+\delta$ there is a value $x_{i}$ of $x$ such that for every $N_{i}^{\prime \prime}>N^{\prime}(i, \delta, N)$

$$
\left|\sum_{n=0}^{N^{\prime}(i, \delta, N)} U_{n}\left(x_{i}^{\prime}\right)-f\left(x_{i}^{\prime}\right)\right| \geqq\left|\sum_{n=0}^{N_{i}^{\prime \prime}} U_{n}(x)-f(x)\right|
$$

for every value of $x$ on the interval $x_{i}-\delta, x_{i}-\delta$ which also lies on $a b . \dagger$

Proof: (1). The condition is necessary. (In the proof $N^{\prime}(i$, $\delta, N)$ is written $N_{i}^{\prime}$.)

By hypothesis $\sum_{n=0}^{\infty} U_{n}(x)$ is uniformly convergent on $a b$ and hence on every subinterval $x_{i}-\delta, x_{i}+\delta$ which is contained in $a b$. Two cases are possible:

(a) $U_{n}(x) \equiv 0$ for every $x$ on $x_{i}-\delta, x_{i}+\delta$ and for every value of $n$ greater than some fixed number $N_{i}$. In this case the conclusion of the proposition holds for $x_{i}{ }^{\prime}$ any $x$ on $x_{i}-\delta$, $x_{i}+\delta$, the two differences

$$
\sum_{n=0}^{N_{i^{\prime}}} U_{n}\left(x_{i}^{\prime}\right)-f\left(x_{i}^{\prime}\right) \text { and } \sum_{n=0}^{N_{i}^{\prime \prime}} U_{n}(x)-f(x)
$$

being zero.

(b) There is no number $N_{i}^{\prime}$ such that for every $n>N_{i}^{\prime}$ $U_{n}(x) \equiv 0$ for $x$ on $x_{i}-\delta, x_{i}+\delta$.

In this case for every $N_{i}{ }^{\prime}$ there are values of $n, n^{\prime}>N_{i}{ }^{\prime}$ and values $x_{i}$ of $x$ on $x_{i}-\delta, x_{i}+\delta$ such that

* The subscripts $i, \delta, N$ indicate that $N^{\prime}(i, \delta, N)$ depends on all of these.

$\dagger \mathrm{A}$ special case of this lemma when

$$
\sum_{n=0}^{\infty}\left|U_{n}(x)\right|
$$

is convergent is given for the sufficient condition by Pringsheim, Mathematische Annalen, vol. 44 (1893), p. 82, and by Bôcher, BuLletis, vol. 4 (1898), p. 368. The theorem of the present paper, besides stating a necessary as well as a sufficient condition, covers a larger class of functions than is covered by the theorem of Pringsheim. Thus the uniform convergence over a certain interval of an alternating series representing $a$ continuous function follows as a special case provided that for every value $x_{i}$ of $x$ of the interval there is a neighborhood of $x_{i}$ on which the numerical values of the terms decrease after a certain term. 


$$
\left|\sum_{n=0}^{n^{\prime}} U_{n}\left(\overline{x_{i}}\right)-f\left(\overline{x_{i}}\right)\right|>2 \epsilon,
$$

where $\epsilon$ is some positive number.

The series $\sum_{n=0}^{\infty} U_{n}(x)$ being uniformly convergent on $x_{i}-\delta$, $x_{i}+\delta$, there is a value of $n, n^{\prime \prime}$ such that for all values of $n$, $\bar{n}>n^{\prime \prime}$,

$$
\left|\sum_{n=0}^{\bar{n}} U_{n}(x)-f(x)\right|<\epsilon .
$$

Since for any value of $k, \sum_{n=0}^{k} U_{n}(x)$ and $f(x)$ are both continuous functions on $x_{i}-\delta, x_{i}+\delta$ there is a definite value of $x$ for which

$$
\left|\sum_{n=0}^{k} U_{n}(x)-f(x)\right|
$$

reaches a maximum on the closed interval $x_{i}-\delta, x_{i}+\delta$. Denote this maximum by $D(k)$. Since $n^{\prime \prime}-n^{\prime}$ is a finite number, there is a value of $n, n=N_{i}^{\prime}, n^{\prime} \leqq N_{i}^{\prime} \leqq n^{\prime \prime}$ for which $D(k)$ reaches the maximum for the values of $k, n^{\prime} \leqq k \leqq n^{\prime \prime}$.

Let $x_{i}^{\prime}$ be a value of $x$ for which

$$
\left|\sum_{n=0}^{N_{i^{\prime}}} U_{n}(x)-f(x)\right|
$$

reaches its maximum. Then by (1) and (2)

$$
\left|\sum_{n=0}^{N_{i}^{\prime}} U_{n}\left(x_{i}^{\prime}\right)-f\left(x_{i}^{\prime}\right)\right| \geqq\left|\sum_{n=0}^{N_{i}^{\prime \prime}} U_{n}(x)-f(x)\right|
$$

for every $x$ on $x_{i}-\delta, x_{i}+\delta$ and every $N_{i}^{\prime \prime}>N_{i}^{\prime}$, which proves the condition necessary.

(2) The condition is sufficient. Since by hypothesis

$$
\sum_{n=0}^{\infty} U_{n}(x)=f(x)
$$

the function $f(x)$ being defined on $a b$, it follows that $\sum_{n=0}^{\infty} U_{n}(x)$ is convergent for every value of $x$ on this interval. Hence for any preassigned positive number $\epsilon$ and any value $x_{i}$ of $x$ on $a b$ 
there is an integer $N_{i}$ such that

$$
\left|\sum_{n=0}^{N_{i}{ }^{\prime}} U_{n}\left(x_{i}\right)-f\left(x_{i}\right)\right|<\epsilon
$$

for every $N_{i}^{\prime} \geqq N_{i}$.

The function $\sum_{n=0}^{N_{i}{ }^{\prime}}\left(U_{n}(x)\right.$ being continuous in the neighborhood of $x=x_{i}$ for every value of $N_{i}{ }^{\prime}$, there is a positive number $\delta^{\prime}$, depending on $\epsilon, x_{i}$, and $N_{i}^{\prime}$, such that for $\left|x-x_{i}\right|<\delta^{\prime}$

$$
\left|\sum_{n=0}^{N_{i}^{\prime}} U_{n}(x)-\sum_{n=0}^{N_{i}^{\prime}} U_{n}\left(x_{i}\right)\right|<\epsilon
$$

for any preassigned $N_{i}^{\prime}>N_{i}$.

Since $f(x)$ is continuous for $x=x_{i}$, there is a $\delta^{\prime \prime}$ such that for $\left|x-x_{i}\right|<\delta^{\prime \prime}$

$$
\left|f(x)-f\left(x_{i}\right)\right|<\epsilon .
$$

Let $\delta^{\prime \prime \prime}$ be the smaller of $\delta^{\prime}$ and $\delta^{\prime \prime}$. Then for $x$ such that $\left|x-x_{i}\right|<\delta^{\prime \prime \prime}$ we have from (3), (4), (5)

$$
\left|\sum_{n=0}^{N_{i}^{\prime}} U_{n}(x)-f(x)\right|<3 \epsilon
$$

for any arbitrarily assigned $N_{i}^{\prime}$.

But by hypothesis, for every $x_{i}$ and an arbitrary $N_{i}$ there is an $N_{i}^{\prime}$ such that for any $N_{i}^{\prime \prime}>N_{i}^{\prime}$ and for $x$ on some interval $x_{i}-\delta^{\prime \prime \prime \prime}, x_{i}+\delta^{\prime \prime \prime \prime}$ there is an $x_{i}{ }^{\prime}$ such that

$$
\left|\sum_{n=0}^{N_{i}^{\prime}} U_{n}\left(x_{i}^{\prime}\right)-f\left(x_{i}^{\prime}\right)\right| \geqq\left|\sum_{n=0}^{N_{i}^{\prime \prime}} U_{n}(x)-f(x)\right| .
$$

Let $\delta$ be the smaller of the two numbers $\delta^{\prime \prime \prime}$ and $\delta^{\prime \prime \prime \prime}$. Since (6) holds for any value of $x$ on $x_{i}-\delta, x_{i}+\delta^{\prime}$ it holds for $x=x_{i}^{\prime}$. Hence if the $N_{i}^{\prime}$ of (7) is chosen as the $N_{i}^{\prime}$ of (6) it follows from (7) that for every $N_{i}^{\prime \prime}>N_{i}^{\prime}$

$$
\left|\sum_{n=0}^{N_{i}^{\prime \prime}} U_{n}(x)-f(x)\right|<3 \epsilon,
$$

provided $\left|x-x_{i}^{\prime}\right| \delta$. That is $\sum_{n=0}^{\infty} U_{n}(x)$ is uniformly conver- 
gent on the interval $x-\delta, x+\delta$, or on that part of such interval which is an $a b$. In this manner for every point $x_{i}$ on $a b$ we obtain an interval of which $x_{i}$ is an internal point (unless $x_{i}$ is $a$ or $b$, in which case $x_{i}$ is the end point of such interval) on which $\sum_{n=0}^{\infty} U_{n}(x)$ is uniformly convergent.

By the Heine-Borel theorem* there is a finite subset of these intervals which completely covers the interval $a b$. Hence it follows by obvious considerations that $\sum_{n=0}^{\infty} U_{n}(x)$ is uniformly continuous on the interval $a b$.

Columbia UnIversity,

February 1, 1911.

\section{PROJECTIVE GEOMETRY.}

Projective Geometry. By Oswald Veblen and John Wesley Young. Volume I. Ginn and Company, Boston, 1910. $\mathrm{x}+342 \mathrm{pp}$.

IN discussing this book, two facts must be constantly borne in mind: the authors knew exactly what they wanted to do, and they were entirely competent to do it. Here is no question of a slovenly work, compiled with no visible object, differing from an honorable line of progenitors only in the smallest details. The book is eminently one with a consistent purpose. Agree with the authors you may not; but if you do not fully enter into their point of view, you will come off second best in the argument.

The authors' main object is to build up projective geometry upon a system of independent axioms. Such a task is, certainly, not new. The names of Pieri, Vahlen, Schur, and others suggest themselves immediately when the question of projective assumptions is raised. Nor is there even any novelty in writing a students' textbook which starts from the ground. We have such a book already in the beautiful treatise of Enriques, and, in fact, we may almost say that this is the

* This theorem may be stated as follows: If on an interval $a b$ there is a set of intervals $[\sigma]$ such that (1) the points $a$ and $b$ are end points of intervals of the set $[\sigma] ;(2)$ every point of the interval $a b$ lies within at least one interval of $[\sigma]$; then there is a finite subset $\sigma_{1}, \ldots, \sigma_{k}$ of the set $[\sigma]$ which satisfy (1) and (2) of the hypothesis. 